\section{NP-016 EPIDEMIOLOGY AND RESISTANCE MAP TO AID ANTIBIOTIC GUIDELINE DEVELOPMENT SINGLE CENTRE RETROSPECTIVE STUDY FROM AN EMERGENCY DEPARTMENT}

\begin{abstract}
1,2,3Ria Benkő*, ${ }^{4}$ Márió Gajdács, 1,2Mária Matuz, ${ }^{2}$ Gabriella Bodó, ${ }^{5}$ Andrea Lázár, ${ }^{6}$ Edit Hajdú, ${ }^{6}$ Erika Papfalvi, ${ }^{3}$ Péter Hannauer, ${ }^{3}$ Péter Erdélyi, ${ }^{3}$ Zoltán Petö. ${ }^{1}$ Department of Clinical Pharmacy, University of Szeged; Szeged; ${ }^{2}$ Central Pharmacy Department, Albert Szent-Györgyi Health Center, Szeged; ${ }^{3}$ Department of Emergency Medicine, Albert SzentGyörgyi Health Center; Szeged; ${ }^{4}$ Department of Pharmacodynamics and Biopharmacy, University of Szeged, Szeged; ${ }^{5}$ Institute of Clinical Microbiology, Albert Szent-Györgyi Health Center; Szeged; ${ }^{6}$ Infectious Disease Ward, 1; st Department of Internal Medicine, Albert Szent-Györgyi Health Center, Szeged
\end{abstract}

10.1136/ejhpharm-2021-eahpconf.363

Background and importance Antimicrobial resistance (AMR) is one of the most important global threats and antibiotic (mis) use is one of the main drivers behind it. Previously we identified suboptimal antibiotic use at our emergency department. International antibiotic guidelines for specific conditions cannot be adopted without the knowledge of local epidemiology and resistance, these are the essential first steps to develop an empiric antibiotic protocol.

Aim and objectives To identify predominant isolates, clinical specimens and reveal resistance patterns of bacterial isolates.

Materials and methods The study was performed at the emergency department of the University of Szeged. All positive microbiological isolates were retrieved for a five-year period. Non-bacterial isolates, contaminants and duplicate isolates were screened and excluded. Bacterial identification was performed using MALDI-TOF MS. Antibiotic susceptibility-testing and interpretation of drug resistance were based on ESCMID/ EUCAST standards.

Results Finally, 6885 bacterial isolates were recovered of which nearly two-thirds ( $\mathrm{n}=4502$ isolates) were Gram-negative species. Blood culture (35.6\%), urinary samples (urinary catheter-23.1\%, midstream urine-12.1\% or deep wound or abscess samples (14.0\%) were the most frequent clinical specimens. The Escherichia genus $(n=2194)$ was the most frequent Gram-negative isolate (with one exception E. coli), followed by members of the Klebsiella genus ( $n=664$, most frequently K. pneumoniae) and the Proteus spp. ( $\mathrm{n}=526$, most frequently $P$. mirabilis). Staphylococcus aureus $(n=561)$, Enterococcus spp. $(\mathrm{n}=471)$ and Streptococcus pneumoniae $(n=431)$ were the most frequent Gram-positive isolates. The top three Gram-positive and Gram-negative isolates were responsible for nearly $75 \%-75 \%$ of the corresponding clinical isolates. The ciprofloxacin resistance were above 30\% for E.coli and K.pneumoniae and were above $40 \%$ for P. mirabilis. On the other hand E.coli was susceptible in acceptable rate (below 20\%) for many beta-lactams. For gram positives vancomycin resistant E. faecium (VRE) was detected in $33.3 \%$, while methicillin resistant $S$. aureus (MRSA) was detected in $16.1 \%$.

Conclusion and relevance The most frequent bacteria and current resistance patterns were identified. Some of the revealed resistance patterns (eg, high fluoroquinolone resistance among Gram-negative bacteria) may pose therapeutic challenges. The results of this survey will guide the development of our local antibiotic guideline.

\section{NP-017 \\ RISK ASSESSMENT OF OCCUPATIONAL EXPOSURE TO CYTOTOXIC DRUGS IN AN OUTPATIENT HOSPITAL PHARMACY}

J Pereira, R Mateus*, S Pires, S Sernache, H Gonçalves, A Gouveia. Instituto Português de Oncologia de Lisboa Francisco Gentil E.P.E., Hospital Pharmacy Services, Lisbon, Portugal

\subsection{6/ejhpharm-2021-eahpconf.364}

Background and importance Safe handling and dispensing of cytotoxic drugs in outpatient setting are of growing concern in hospital pharmacy. However, surface contamination studies mainly focus on the preparation areas. As oral therapy gains significance, it is critical to assess its role as a potential source of contamination in non-compounding environments.

Aim and objectives a) Workflow analysis for critical sample sites selection; b) Designing of a risk matrix considering the contamination level $(\mathrm{ng} / \mathrm{cm} 2)$; c) Implementing corrective measures and sampling frequency according to the risk level.

Materials and methods Drugs selected were 6-Mercaptopurine (6-MP) and Capecitabine (CPC), based on potential risk for the operator (number of dispensations vs. handling level) and 4 critical areas (storage drawer; hood; repackaging bench; dispensing counter). Samples were collected by wipe sampling and sent to analysis by LC-MS/MS, in a certified laboratory (IUTA). ${ }^{1}$ Two sampling periods were carried out, interposed with corrective measures.

Results Out of five samples collected, two presented results above the reference value of $0.1 \mathrm{ng} / \mathrm{cm}^{2: 1}$ storage drawer (CPC) and hood (6-MP). Following a cleaning procedure in the storage drawer (CPC), a value of $0.014 \mathrm{ng} / \mathrm{cm}^{2}$ was obtained. The remaining results were below the assay's limit of quantification (LoQ).

Conclusions and relevance The storage areas were identified as of increased risk. However, the limited number of samples conditioned the total mapping of critical areas.

The results analysis led to the definition of risk levels: low risk<LoQ; LoQ $>$ medium risk $<0.1 \mathrm{ng} / \mathrm{cm}^{2}$; high risk $>0.1 \mathrm{ng} / \mathrm{cm}^{2}$. It is intended to extend the sampling to all dispensing pathways. The development and evaluation of corrective measures and sampling frequency is still pending on future results, although the cleaning methodology has proven to be effective.

There is evidence of contamination from handling oral formulations. The lack of risk perception leads to the undervaluing of routine procedures, such as cleaning, enhancing occupational exposure.

This project aims to ensure that the occupational exposure level is reduced to a value as low as technically possible.

\section{REFERENCES AND/OR ACKNOWLEDGEMENTS}

1. Kiffmeyer TK, et al. Application and assessment of a regular environmental monitoring of the antineoplastic drug contamination level in pharmacies-the MEWIP project. Ann Occup Hyg 2013 May;57(4):444-55. 